$\mathrm{DFPD} / 92 / \mathrm{TH} / 36$

\title{
The Electroweak Phase Transition in a Nonminimal Supersymmetric Model
}

\author{
Massimo Pietroni \\ Dipartimento di Fisica "Galileo Galilei", Università \\ di Padova, Via F.Marzolo 8, 35131 Padova, Italia
}

\begin{abstract}
We study the electroweak phase transition in a supersymmetric version of the Standard Model, in which a gauge singlet superfield is added to the Higgs sector. We show that the order of the transition is determined by the trilinear soft supersymmetry breaking terms rather than by the $O\left(m^{3} T\right)$ term in the 1-loop, $T \neq 0$ corrections. This fact removes the Standard Model upper bound on the Higgs mass, $m_{H}<55 \mathrm{GeV}$, coming from the requirement that baryon asymmetry is not washed out by anomalous electroweak processes. We perform a numerical analysis of parameter space including in the effective potential top-stop contribution to 1-loop radiative corrections. We find that this model is compatible with the preservation of baryon asymmetry for masses of the lightest scalar up to about $170 \mathrm{GeV}$.
\end{abstract}




\section{Introduction.}

Several years ago Sakharov [1] realized that the observed baryon asymmetry of the Universe (BAU) could have been generated at some early stage of cosmic evolution at which three conditions were fulfilled: B-violating interactions, $\mathrm{C}$ and $\mathrm{CP}$ violation, and a departure from thermodynamic equilibrium. All these conditions are fulfilled in grand unification theories (GUT), in which the baryon asymmetry is generated in the out-of-equilibrium, B-violating, decay of some superheavy boson [2]. However this scenario presents severe complications. First of all, as noted by Bocharev, Kuzmin and Shaposhnikov[3], any nonzero fermion number $(\mathrm{B}+\mathrm{L})$ created at the GUT epoch is almost completely erased [4] by the anomalous electroweak $\mathrm{B}+\mathrm{L}$-violating processes [5], which are in equilibrium down to a temperature $T \sim 10^{2} \mathrm{GeV}$. So, if no B-L is created at the GUT phase transition, then no baryon excess survives down to the Fermi scale. Moreover, if B-L-violating interactions $(\delta L=2$ Majorana masses for neutrinos, R-parity violating interactions in SUSY models [5]...) are present and in equilibrium at high $(T \gg 100 \mathrm{GeV})$ temperature, then also an eventual B-L component of the GUT-generated BAU vanishes before the onset of the electroweak era.

The above considerations led many people to investigate the possibility that the BAU was generated at the electroweak phase transition,([6]-[13]). Here the difficulty is threefold; first, $\mathrm{CP}$ violation in the Standard Model is too small [7], second, it is not obvious that the transition is of first order strongly enough to make the baryon number production effective and, third, we must avoid the wiping out of the baryon asymmetry by the anomalous processes. The efficiency of the latter is suppressed by the exponential of (minus) the 'sphaleron' mass [6] over the temperature

$$
\frac{E^{s p h}(T)}{T}=\frac{4 \pi v(T)}{g_{W}} \frac{B}{T},
$$

where $B\left(\frac{\lambda}{g_{W}^{2}}\right)$ is a slowly varying function of the couplings $B(0)=1.56$, $B(\infty)=2.72$, and $v(T)$ the value of the vacuum expectation value (VEV) of the Higgs field at the temperature T. The requirement that (1) is sufficiently large at the phase transition gives a lower bound [6] on the value of the vacuum expectation value (VEV) at the critical temperature (which we define 
here as the temperature at which the effective potential becomes flat at the origin, $\left.V^{\prime \prime}(0)=0\right)$

$$
\frac{v\left(T_{C}\right)}{T_{C}} \gtrsim 1.3 .
$$

In the Standard Model $v\left(T_{C}\right) / T_{C}$ is given by the ratio of the coefficient of the term cubic in the Higgs field, $\delta$, and the quartic self-coupling, $\lambda$. So it is easy to see that (2) imposes an upper bound on the Higgs mass

$$
\left(\frac{M_{H}}{246.2 G e V}\right)^{2} \lesssim 2.3 \delta .
$$

The crucial $\delta$ coefficient is zero in the Standard Model at the tree level; it is generated by the one-loop finite temperature corrections $[14,15]$ as a subleading term in the $M / T$ expansion [15], and its evaluation requires a careful resummation of the leading infrared (IR) divergencies [14,16]. Despite all the uncertainties related to this procedure, it seems by now established that the Standard Model bound (3) has been ruled out by LEP results, $M_{H}>57 \mathrm{GeV}$ [17], or it will be in the near future.

Since $\delta$ receives contribution by the bosonic degrees of freedom with masses lighter than the temperatures in consideration Q], attempts have been made to relax the bound on $M_{H}$ by considering the Standard Model with an extended Higgs sector $[6,8,9,18]$, or minimal SUSY Standard Model (MSSM), in which two higgs doublets are present [10]. In all these cases the cubic term is again a one-loop effect and its evaluation meets the same IR problems that we find in the Standard Model. Moreover, as discussed in ref. [13], there is a large suppression $\left(O\left(\alpha_{W}^{8}\right)\right)$ of the baryon asymmetry in models containing only scalar doublets, without extra CP violation sources than the Higgs potential and the Yukawa couplings.

In this paper we study the electroweak phase transition in the simplest possible extension of the MSSM with soft supersymmetry breaking, in which a gauge singlet superfield is added to the superpotential $[19,20]$. From the point of view of the phase transition the main feature of this model is the occurrence of trilinear soft SUSY breaking terms in the Higgs sector of the tree level effective potential. These terms behave as effective cubic terms in the radial direction from the origin of the field space to the vacuum, so that the

\footnotetext{
${ }^{1}$ in fact it may be shown numerically that the $M / T$ expansion is a good approximation up to $\frac{M}{T} \sim 2.2[9]$
} 
potential has a barrier along this direction already at the tree-level. Moreover, including the leading (no IR problem) finite temperature corrections, at one-loop, we get also positive linear terms which enhance the barrier between the false and the true vacuum. As a result, this model leads very naturally to a strongly first order phase transition and, as we will see, the bound (2) may be fulfilled in a large portion of the parameter space corresponding to values of the mass of the lighter scalar up to about $170 \mathrm{GeV}$.

\section{The model.}

We assume [20] that the squark and slepton fields have vanishing vacuum expectation values so that we can restrict our attention to the Higgs sector of the superpotential involving the superfields $\tilde{H}_{1}, \tilde{H}_{2}$ and $\tilde{N}$ only

$$
W_{\text {Higgs }}=\lambda \tilde{H}_{1} \tilde{H}_{2} \tilde{N}-\frac{1}{3} k \tilde{N}^{3} .
$$

The tree level scalar potential is given by

$$
\begin{aligned}
& V= V_{F}+V_{D}+V_{\text {Soft }} ; \\
& V_{F}=|\lambda|^{2}\left[|N|^{2}\left(\left|H_{1}\right|^{2}+\left|H_{2}\right|^{2}\right)+\left|H_{1} H_{2}\right|^{2}\right]+k^{2}|N|^{4}-\left(\lambda k^{*} H_{1} H_{2} N^{2 *}+h . c\right) ; \\
& V_{D}= \frac{g^{2}+g^{\prime 2}}{8}\left(\left|H_{2}\right|^{2}-\left|H_{1}\right|^{2}\right)^{2}+\frac{g^{2}}{2}\left|H_{1}^{\dagger} H_{2}\right|^{2} ; \\
& V_{\text {Soft }}= m_{H_{1}}^{2}\left|H_{1}\right|^{2}+m_{H_{2}}^{2}\left|H_{2}\right|^{2}+m_{N}^{2}|N|^{2} \\
&-\left(\lambda A_{\lambda} H_{1} H_{2} N+\text { h.c. }\right)-\left(\frac{1}{3} k A_{k} N^{3}+\text { h.c. }\right), \\
& \text { where } H_{1} \equiv\left(\begin{array}{c}
H_{1}^{0} \\
H^{-}
\end{array}\right), H_{2} \equiv\left(\begin{array}{c}
H^{+} \\
H_{2}^{0}
\end{array}\right) .
\end{aligned}
$$

Note in particular the presence of trilinear terms in $V_{\text {Soft }}$, which are absent in the Higgs sector of the MSSM.

Redefining the global phases of $H_{1}$ and $N$ we can always take $\lambda A_{\lambda}$ and $k A_{k}$ real and positive, while by an $\mathrm{SU}(2) \mathrm{XU}(1)$ global rotation we put

$$
v^{+} \equiv<H^{+}>=0, v_{2} \equiv<H_{2}^{0}>\in R^{+} .
$$


We will not discuss the $\mathrm{CP}$ violation aspect of the BAU generation problem, so we assume CP conservation. Choosing $\lambda k>0$ we avoid explicit CP violation and find three degenerate minima (note that the problem has a discrete $Z_{3}$ symmetry[21]) corresponding to different values for $\phi_{1}$ and $\phi_{x}$, which are respectively the phases of $v_{1} \equiv<H_{1}>$ and $x \equiv<N>$

$$
\left\{\begin{array}{l}
\phi_{1}=0, \phi_{x}=0 \\
\phi_{1}=\frac{2}{3} \pi, \phi_{x}=\frac{4}{3} \pi \\
\phi_{1}=\frac{4}{3} \pi, \phi_{x}=\frac{2}{3} \pi
\end{array}\right.
$$

Due to our assumption of CP conservation, the true vacuum of the theory is the one with both phases equal to zero.

We will work in the unitary gauge

$$
H_{1}=\left(\begin{array}{c}
\left(R e H_{1}^{0}+\frac{i}{\sqrt{2}} \sin \beta A^{0}\right) \\
\sin \beta C^{+*}
\end{array}\right), H_{2}=\left(\begin{array}{c}
\cos \beta C^{+} \\
\left(R e H_{2}^{0}+\frac{i}{\sqrt{2}} \cos \beta A^{0}\right)
\end{array}\right)
$$

where, as usual, $\operatorname{tg} \beta=v_{2} / v_{1}$, and

$$
\begin{aligned}
A^{0} & \equiv \sqrt{2}\left(\sin \beta \operatorname{Im} H_{1}^{0}+\cos \beta \operatorname{Im} H_{2}^{0}\right) ; \\
C^{+} & \equiv \cos \beta H^{+}+\sin \beta H^{-*} .
\end{aligned}
$$

The combinations orthogonal to $C^{+}$and $A^{0}$ are the would-be Goldstone bosons giving masses to the $W^{ \pm}$and $Z^{0}$. The physical degrees of freedom of the model are given by three scalars, two pseudoscalars and one charged scalar.

Before proceeding in the calculation of the finite temperature corrections, we wish to comment on some aspects of the tree level potential which will be used in what follows. First, it contains seven free parameters: $\lambda, k, A_{\lambda}, A_{k}$, $m_{H_{1}}^{2}, m_{H_{2}}^{2}$ and $m_{N}^{2}$. Imposing the stationarity conditions in $\left(H_{1}^{0}, H_{2}^{0}, N\right)=$ $\left(v_{1}, v_{2}, x\right)$ with the constraint

$$
v_{1}^{2}+v_{2}^{2} \equiv v^{2}=(246.2 G e V)^{2},
$$

we express the soft masses in terms of the six parameters $\lambda, k, A_{\lambda}, A_{k}, \operatorname{tg} \beta$ 
and $x$

$$
\left\{\begin{aligned}
m_{H_{1}}^{2} & =\lambda\left(A_{\lambda}+k x\right) x t g \beta-\lambda^{2}\left(x^{2}+v^{2} \sin ^{2} \beta\right) \\
& -\frac{g^{2}+g^{\prime 2}}{4} v^{2} \cos 2 \beta \\
m_{H_{2}}^{2} & =\lambda\left(A_{\lambda}+k x\right) x \operatorname{cotg} \beta-\lambda^{2}\left(x^{2}+v^{2} \cos ^{2} \beta\right) \\
& +\frac{g^{2}+g^{\prime 2}}{4} v^{2} \cos 2 \beta \\
m_{N}^{2} & =\lambda A_{\lambda} \frac{v^{2}}{2 x} \sin 2 \beta+k A_{k} x-\lambda^{2} v^{2}-2 k^{2} x^{2} \\
& +\lambda k v^{2} \sin 2 \beta
\end{aligned}\right.
$$

Note that (6) does not guarantee that $\left(v_{1}, v_{2}, x\right)$ is the global minimum of the effective potential (4); for each choice of the parameters we must verify that this is indeed the case.

In addition, we have to be sure that $v^{-}$vanishes in the vacuum; looking at the potential along the charged direction,

$$
\begin{aligned}
V_{c h}= & \left|H^{-}\right|^{2}\left[m_{H_{1}}^{2}+\lambda^{2}|N|^{2}+\frac{g^{2}}{4}\left(\left|H_{1}\right|^{2}+\left|H_{2}\right|^{2}\right)+\right. \\
& \left.\frac{g^{\prime 2}}{4}\left(\left|H_{1}\right|^{2}-\left|H_{2}\right|^{2}\right)\right]+\frac{g^{2}+g^{\prime 2}}{8}\left|H^{-}\right|^{4},
\end{aligned}
$$

we see that a sufficient condition in order to have $v^{-}=0$ as a global minimum is

$$
m_{H_{1}}^{2}+\lambda^{2} x^{2}+\frac{g^{2}}{4} v^{2}+\frac{g^{\prime 2}}{4} v^{2} \cos 2 \beta>0
$$

Now we introduce the finite temperature corrections at the one-loop level. As usual [15] they can be split into $T=0$ and $T \neq 0$ contributions.

The former are given by ([22])

$$
\Delta V_{T=0}^{1-\text { loop }}=\frac{1}{64 \pi^{2}} \operatorname{Str}\left\{M^{4}(\phi)\left[\ln \frac{M^{2}(\phi)}{Q^{2}}-\frac{3}{2}\right]\right\},
$$

where $M^{4}(\phi)$, with $\phi=\left(H_{1}, H_{2}, N\right)$, is the field dependent mass matrix and $Q$ the renormalization point. The $Q^{2}$ dependence in (8) is compensated by that of the renormalized parameters, so that the full effective potential is independent of $Q^{2}$.

We have approximated expression (8) by considering only the top and stop contributions: in addition, we take the two stops' masses to be degenerate

$$
m_{\tilde{t}_{L, R}}^{2} \approx \tilde{m}_{Q}^{2}+h_{t}^{2}\left|H_{2}\right|^{2}, \quad m_{t}^{2}=h_{t}^{2}\left|H_{2}\right|^{2},
$$


where $\tilde{m}_{Q}^{2}$ is the squarks soft mass (we assume $\tilde{m}_{U}^{2}=\tilde{m}_{Q}^{2}$ ) and $h_{t}$ is the Yukawa coupling. We fix the renormalization scale $Q$ at a value $\bar{Q}$ such that the tree level relations (6), expressed in terms of the renormalized parameters, do not change, i.e.

$$
\frac{\partial \Delta V_{T=0}^{1-\text { loop }}}{\partial H_{2}} \mid \begin{aligned}
& Q^{2}=\bar{Q}^{2}=0 . \\
& H_{2}=v_{2}
\end{aligned}
$$

Since we have neglected the $\tilde{t}_{L}-\tilde{t}_{R}$ mixing terms in the stop mass matrix, the correction (8) does not affect the pseudoscalar mass matrix [22], while it modifies the scalar spectrum.

The $T \neq 0$ part of one-loop corrections [15] is given by the expression

$$
\begin{aligned}
\Delta V_{T \neq 0}^{1-l o o p}(\phi, T) & =\sum_{F} \frac{g_{F} T^{4}}{2 \pi^{2}} \int_{0}^{\infty} d x x^{2} \ln \left[1+e^{-\left(x^{2}+\frac{m_{F}^{2}(\phi)}{T^{2}}\right)^{\frac{1}{2}}}\right] \\
& +\sum_{B} \frac{g_{B} T^{4}}{2 \pi^{2}} \int_{0}^{\infty} d x x^{2} \ln \left[1-e^{-\left(x^{2}+\frac{m_{B}^{2}(\phi)}{T^{2}}\right)^{\frac{1}{2}}}\right]
\end{aligned}
$$

where $m_{F(B)}^{2}(\phi)$ is the tree-level mass of a fermion (boson) in presence of the background fields $\phi\left(=H_{1}, H_{2}, N\right), g_{F(B)}$ is the corresponding number of degrees of freedom and the sum runs over all fermions (F) and bosons (B) of the theory. For values of the fields such that $m(\phi) / T<1$ we can expand $(11,12)$ as $([15])$

$$
\begin{aligned}
\Delta V_{T \neq 0}^{1-\text { loop }}(\phi, T) & \approx \sum_{F} g_{F} T^{4}\left[-\frac{7 \pi^{2}}{720}+\frac{m_{F}^{2}(\phi)}{48 T^{2}}+O\left(\frac{m_{F}^{4}(\phi)}{T^{4}} \ln \frac{m_{F}(\phi)}{T}\right)\right] \\
& +\sum_{B} g_{B} T^{4}\left[-\frac{\pi^{2}}{90}+\frac{m_{B}^{2}(\phi)}{24 T^{2}}+O\left(\frac{m_{B}^{3}(\phi)}{T^{3}}\right)\right],
\end{aligned}
$$

where we have neglected the subleading $m_{B}^{3} / T^{3}$ term in the expansion for the bosons. As we said, this term is essential in the Standard Model as in the MSSM in order to obtain a first order phase transition, while in the present model the barrier between the true and the false vacua is given mainly by the tree-level trilinear terms. We have checked numerically that the cubic term coming from one-loop corrections changes the values of $T_{C}$ and $V\left(T_{C}\right)$ only by a few percent. 
The degrees of freedom corresponding to masses $m>T$ are Boltzmannsuppressed and in this limit the correction (15) reduces to $([9])$

$$
\Delta V_{T \neq 0}^{1-l o o p}(\phi, T) \sim \sum_{F, B} \frac{g_{i} T^{2}}{(2 \pi)^{\frac{3}{2}}} m_{i}^{2} \sqrt{\frac{T}{m_{i}}} e^{-\frac{m_{i}}{T}}\left[1+\frac{15}{8} \frac{T}{m_{i}}+O\left(\frac{T^{2}}{m_{i}^{2}}\right)\right] .
$$

Since we are considering temperatures of the order of $M_{W}$ the exponential factor in the previous expression allows us, with good approximation, to neglect SUSY particles with masses above the SUSY threshold which we assume to be

$$
\tilde{m} \approx 1 T e V
$$

Lighter particles will contribute to (13) even if it may happen that $M(\phi) / T \gtrsim$ 1 for some of them, at large values of the field. However (see the footnote n. 1) this does not change matters essentially with respect to the situation in which we use $(11,12)$. Moreover the use of (13) allows us to perform an analytic study of the critical temperature and of the phase transition which can help us to understand the main properties of the model, otherwise hidden by a numerical evaluation of the integrals in $(11,12)$.

In the following we will derive the mass matrix for the relevant degrees of freedom of the theory on the background of all the scalar fields. We will then discuss bounds on the parameter space coming from the requirement of a correct symmetry breaking pattern (i.e. no VEV's for $A^{0}, \operatorname{Im} N$ and for $C^{+}$), and on the LEP limits on chargino masses. Finally we will scan the allowed parameter space, find the values of the critical temperatures, and the minimum of the potential at those temperatures.

\section{The mass matrix.}

Let us consider the matrix of the second derivatives of the tree-level effective potential: due to $\mathrm{C}$ and $\mathrm{CP}$ conservation it is a block-diagonal 6x6 matrix containing the $3 \times 3$ neutral scalars matrix, the $2 \times 2$ pseudoscalar matrix and the charged scalar mass. The elements of these matrices are given by:

a)neutral scalars: 
in the basis $\left(\mathrm{ReH}_{1}, \mathrm{ReH}_{2}, \mathrm{ReN}\right)$ we have

$$
\begin{aligned}
& M_{S 11}^{2}=2 m_{H_{1}}^{2}+2 \lambda^{2}\left(|N|^{2}+\left|H_{2}^{0}\right|^{2}\right) \\
& +\frac{g^{2}+g^{\prime 2}}{2}\left[\operatorname{Re}\left(H_{1}^{0^{2}}\right)+2\left|H_{1}^{0}\right|^{2}-\left|H_{2}^{0}\right|^{2}-\left|H^{+}\right|^{2}+\left|H^{-}\right|^{2}\right]+g^{2}\left|H^{+}\right|^{2} \\
& M_{S 22}^{2}=2 m_{H_{2}}^{2}+2 \lambda^{2}\left(|N|^{2}+\left|H_{1}^{0}\right|^{2}\right) \\
& +\frac{g^{2}+g^{\prime 2}}{2}\left[R e\left(H_{2}^{0^{2}}\right)+2\left|H_{2}^{0}\right|^{2}-\left|H_{1}^{0}\right|^{2}-\left|H^{-}\right|^{2}+\left|H^{+}\right|^{2}\right]+g^{2}\left|H^{-}\right|^{2} \\
& M_{S 33}^{2}=2 m_{N}^{2}+2 \lambda^{2}\left(\left|H_{1}^{0}\right|^{2}+\left|H_{2}^{0}\right|^{2}+\left|H^{+}\right|^{2}+\left|H^{-}\right|^{2}\right)+4 k^{2}\left[3(\operatorname{ReN})^{2}+(\operatorname{ImN})^{2}\right] \\
& \text { - } 4 k A_{k} \operatorname{ReN}-4 \lambda k \operatorname{Re}\left(H_{1} H_{2}\right) \text {; } \\
& M_{S 12}^{2}=\left[4 \lambda^{2}-2\left(g^{2}+g^{\prime 2}\right)\right] R e H_{1}^{0} R e H_{2}^{0}-4 \lambda A_{\lambda} R e N \\
& \text { - } 4 \lambda k\left[(\operatorname{ReN})^{2}-(\operatorname{Im} N)^{2}\right]-4\left(\lambda^{2}-\frac{g^{2}}{2}\right) \operatorname{Re}\left(H^{+} H^{-}\right) \text {; } \\
& M_{S 13}=4 \lambda^{2} \operatorname{Re} H_{1}^{0} \operatorname{ReN}-4 \lambda k R e\left(H_{2}^{0} N^{*}\right)-2 \lambda A_{\lambda} R e H_{2}^{0} \\
& M_{S 23}=4 \lambda^{2} \operatorname{Re} H_{2}^{0} \operatorname{Re} N-4 \lambda k \operatorname{Re}\left(H_{1}^{0} N^{*}\right)-2 \lambda A_{\lambda} \operatorname{Re} H_{1}^{0}
\end{aligned}
$$

b)pseudoscalars:

in the basis $\left(A_{0}, \operatorname{Im} N\right)$

$$
\begin{aligned}
M_{P 11} & =\sin ^{2} \beta\left[m_{H_{1}}^{2}+\lambda^{2}\left(|N|^{2}+\left|H_{2}^{0}\right|^{2}\right)+\frac{g^{2}}{2}\left|H^{+}\right|^{2}\right. \\
& +\frac{g^{2}+g^{\prime 2}}{4}\left(\left(2\left|H_{1}^{0}\right|^{2}-\left|H_{2}^{0}\right|^{2}-\operatorname{Re}\left(H_{1}^{0^{2}}\right)+\left|H^{-}\right|^{2}-\left|H^{+}\right|^{2}\right)\right]
\end{aligned}
$$




$$
\begin{aligned}
M_{P 22} & =m_{N}^{2}++2 \lambda^{2}\left(\left|H_{1}\right|^{2}+\left|H_{2}\right|^{2}\right) \\
& +4 k^{2}\left[2|N|^{2}-\operatorname{Re}\left(N^{2}\right)\right]+4 \lambda k \operatorname{Re}\left(H_{1} H_{2}\right)+4 k A_{k} \operatorname{ReN} ; \\
M_{P 12}= & \frac{1}{\sqrt{2}} \sin \beta\left\{4 \lambda^{2} \operatorname{Im} N \operatorname{Im} H_{1}^{0}-4 \lambda k \operatorname{Re}\left(N^{*} H_{2}^{0}\right)+2 \lambda A_{\lambda} \operatorname{Re} H_{2}^{0}\right\} \\
+ & \frac{1}{\sqrt{2}} \cos \beta\left\{4 \lambda^{2} \operatorname{ImN} \operatorname{Im} H_{2}^{0}-4 \lambda k \operatorname{Re}\left(N^{*} H_{1}^{0}\right)+2 \lambda A_{\lambda} \operatorname{Re} H_{1}^{0}\right\} ;
\end{aligned}
$$

c) charged scalar:

$$
\begin{aligned}
m_{C}^{2} & =\cos ^{2} \beta\left[m_{H_{2}}^{2}+\lambda^{2}\left(|N|^{2}+\left|H^{-}\right|^{2}\right)+\frac{g^{2}-g^{\prime 2}}{4}\left|H_{1}^{0}\right|^{2}\right. \\
& \left.+\frac{g^{2}+g^{\prime 2}}{4}\left(\left|H_{2}^{0}\right|^{2}+2\left|H^{+}\right|^{2}-\left|H^{-}\right|^{2}\right)\right] \\
& +\sin \beta \cos \beta\left[-\lambda^{2}\left(H_{1}^{0} H_{2}^{0}-H^{+} H^{-}\right)-\frac{g^{2}+g^{\prime 2}}{4}\left(H^{+} H^{-}\right)\right. \\
& \left.+\frac{g^{2}}{2}\left(H_{1}^{0} H_{2}^{0}\right)+\lambda k N^{2}+\lambda A_{\lambda} N^{*}+h . c .\right] \\
& +\sin ^{2} \beta\left[m_{H_{1}}^{2}+\lambda^{2}\left(|N|^{2}+\left|H^{+}\right|^{2}\right)+\frac{g^{2}-g^{\prime 2}}{4}\left|H_{2}^{0}\right|^{2}\right. \\
& \left.+\frac{g^{2}+g^{\prime 2}}{4}\left(\left|H_{1}^{0}\right|^{2}+2\left|H^{-}\right|^{2}-\left|H^{+}\right|^{2}\right)\right] \cdot
\end{aligned}
$$

Next we come to the SUSY particles. Among the squarks and leptons we should consider only the stop-quarks $\tilde{t}_{L, R}$, the others having negligible Yukawa couplings. However $\tilde{t}_{L, R}$ masses (9) are dominated by $\tilde{m}_{Q}$, which at the electroweak scale is much greater than $M_{W}$ (its renormalization group equation is dominated by the strong coupling [19]) and then their contribution is Boltzmann-suppressed.

Charginos have a 2x2 mass matrix (in the basis $\left(\tilde{H}^{-}, \tilde{W}^{-}\right)$)

$$
M_{c h}^{2}=\left(\begin{array}{cc}
\lambda N & g \sqrt{2} H_{2}^{0} \\
g \sqrt{2} H_{1}^{0} & M_{2}
\end{array}\right),
$$


where $M_{2}$ is the gaugino direct mass term. Assuming

$$
M_{2} \approx \tilde{m} \gg M_{W} \approx \lambda x
$$

we get

$$
m_{c h 1}{ }^{2} \approx \lambda^{2}|N|^{2} ; m_{c h 2}{ }^{2} \approx M_{2}^{2}+2 g^{2}\left(\left|H_{1}^{0}\right|^{2}+\left|H_{2}^{0}\right|^{2}\right),
$$

which shows that the lightest chargino may be in thermodynamic equilibrium and contributes to (13). The LEP bound on the lowest chargino mass [23]

$$
m_{\chi^{ \pm}}>45 \mathrm{GeV}
$$

will be imposed to constrain the parameter space for $\lambda$ and $\mathrm{x}$. The $5 \mathrm{x} 5$ neutralinos mass matrix, $M_{N}^{2}$ has two heavy (of order $M_{1,2}$ ) Boltzmann suppressed eigenvalues. With the choice of the parameters that will be illustrated in the following, the off-diagonal terms ${M_{N 12}}^{2}, M_{N 13}{ }^{2}$ and $M_{N 23}{ }^{2}$, are negligible in comparison with the three diagonal ones, $M_{N 11}{ }^{2}, M_{N 22}{ }^{2}$ and $M_{N 33}{ }^{2}$, so that the approximate masses for the three lightest neutralinos are given by the following expressions

$$
\begin{aligned}
& m_{N 1}{ }^{2} \approx \lambda^{2}\left(|N|^{2}+\left|H_{2}^{0}\right|^{2}\right)+\left(g^{2}+g^{\prime 2}\right)\left|H_{1}^{0}\right|^{2} ; \\
& m_{N 2}{ }^{2} \approx \lambda^{2}\left(|N|^{2}+\left|H_{1}^{0}\right|^{2}\right)+\left(g^{2}+g^{\prime 2}\right)\left|H_{2}^{0}\right|^{2} ; \\
& m_{N 3}{ }^{2} \approx \lambda^{2}\left(\left|H_{1}^{0}\right|^{2}+\left|H_{2}^{0}\right|^{2}\right)+4 k^{2}|N|^{2} \text {. }
\end{aligned}
$$

Now we are ready to write the one-loop, $T \neq 0$, correction in the high temperature limit. It is given by

$$
\begin{aligned}
\Delta V_{T \neq 0}^{1-\text { loop }}(\phi, T) & =\frac{T^{2}}{24}\left[\operatorname{Tr} M_{S}^{2}+\operatorname{Tr} M_{P}^{2}+2 m_{C}^{2}+6 M_{W}^{2}+3 M_{Z}^{2}\right. \\
& \left.+6 m_{t}^{2}+2 m_{c h 1}^{2}+m_{N 1}^{2}+m_{N 2}^{2}+m_{N 3}^{2}\right]^{\prime},
\end{aligned}
$$

where $[\cdots]^{\prime}$ means that the sum runs on the light masses only. As the fielddependent mass of a given particle becomes much greater than the temperature, the contribution of that particle to the previous expression is switched off. Numerical evaluations [9] of the integrals in $(11,12)$ show that we can approximate them at better than ten percent using expression (13) for masses up to $m / T \sim 2$. 


\section{Determination of the critical temperature.}

We defined the critical temperature $T_{C}$ as that value of $\mathrm{T}$ at which the origin of the field space becomes a saddle point for the effective potential. In fact, it is well known $[9,24]$ that the transition proceeds by quantum tunnelling and is completed at a temperature higher than $T_{C}$, however we are now interested in determining the order of the transition, which is parameterized by the vacuum expectation value at $T_{C}\left(v\left(T_{C}\right)=0\right.$ means a second order phase transition, $v\left(T_{C}\right) \neq 0$ a first order one).

It is easy to check that at high temperature symmetry is restored, in the sense that the origin is a minimum of the effective potential.

The critical temperature is then defined by the condition

$$
\left.\operatorname{det}\left[M_{S}^{T^{2}}\left(T_{C}\right)\right]\right|_{\phi_{i}=0}=0
$$

where the effective mass matrix $M_{S}^{T}$ is given by the second derivatives of the full one-loop potential at finite temperature with respect to the scalar fields. In the origin of the field space the effective mass matrix is approximatively diagonal in the basis $\left(\mathrm{ReH}_{1}, \mathrm{ReH}_{2}, \mathrm{ReN}\right)$

$$
\left.M_{S}^{T^{2}}\left(T_{C}\right)\right|_{\phi_{i}=0}=2\left(\begin{array}{ccc}
m_{H_{1}}^{2}+\frac{T^{2}}{24} C_{H_{1}} & \frac{T^{2}}{24} C_{12} & 0 \\
\frac{T^{2}}{24} C_{12} & m_{H_{2}}^{2}+\frac{T^{2}}{24} C_{H_{2}}+\Delta_{r a d} & 0 \\
0 & 0 & m_{N}^{2}+\frac{T^{2}}{24} C_{N}
\end{array}\right),
$$

where

$$
\begin{aligned}
C_{H_{1}} & =\lambda^{2}\left(5+\cos ^{2} \beta\right)+\frac{g^{2}}{4}(22-\cos 2 \beta)+\frac{g^{\prime 2}}{4}(8-3 \cos 2 \beta) \\
C_{H_{2}} & =6 h_{t}^{2}+\lambda^{2}\left(5+\sin ^{2} \beta\right)+\frac{g^{2}}{4}(22+\cos 2 \beta)+\frac{g^{\prime 2}}{4}(8+3 \cos 2 \beta) \\
C_{12} & =\sin 2 \beta\left(\frac{g^{2}}{2}-\lambda^{2}\right) \\
C_{N} & =9 \lambda^{2}+12 k^{2}+3 \lambda k \sin 2 \beta
\end{aligned}
$$

and

$$
\Delta_{\text {rad }}=\frac{3 h_{t}^{2}}{8 \pi^{2}} \tilde{m}^{2} \log \frac{\tilde{m}^{2}}{\tilde{m}^{2}+m_{t}^{2}}
$$


Neglecting the off-diagonal term, the critical temperature is given by the highest among $T_{H_{1}}^{S}, T_{H_{2}}^{S}$ and $T_{N}^{S}$ where

$$
T_{i}^{S^{2}}=-\frac{24 m_{i}^{2}+\delta_{i, H_{2}} \Delta_{r a d}}{C_{i}} \quad\left(i=H_{1}, H_{2}, N\right) .
$$

Obviously the effective potential can become flat only along those directions corresponding to negative soft masses. Negative values of $m_{N}^{2}$ may however be dangerous for $\mathrm{CP}$ conservation, which we have assumed as a 'boundary condition' for the vacuum at $\mathrm{T}=0$; if we look at the effective mass matrix for pseudoscalars in the origin of field space, and in the basis $\left(A^{0}, \operatorname{Im} N\right)$, we get

$$
M_{P}^{T}{ }^{2}=2\left(\begin{array}{cc}
m_{H_{1}}^{2} \sin ^{2} \beta+m_{H_{2}}^{2} \cos ^{2} \beta+\frac{T^{2}}{24} P_{A^{0}} & 0 \\
0 & m_{N}^{2}+\frac{T^{2}}{24} P_{N}
\end{array}\right)
$$

where

$$
\begin{aligned}
P_{A^{0}} & =\lambda^{2}\left(3+\frac{5}{2} \sin ^{2} 2 \beta\right)+\frac{5}{16}\left(g^{2}+g^{2}\right) \cos ^{2} 2 \beta \\
P_{N} & =9 \lambda^{2}+12 k^{2}-3 \lambda k \sin 2 \beta
\end{aligned}
$$

Then, at the temperature

$$
T_{N}^{P^{2}}=-\frac{24 m_{N}^{2}}{P_{N}} \geq T_{N}^{S^{2}}
$$

the potential becomes flat, in the origin, along the direction $\operatorname{Im} N$, while it remains convex in the direction $\operatorname{ReN}$. So, if at that temperature the transition in the scalar fields has not yet taken place then the imaginary part of the fields acquires a vacuum expectation value, and, when the temperature goes to zero, the vacuum is no more $\phi_{1}=\phi_{x}=0$ (see eq.(5)).

Due to the heavy top [19], $m_{H_{2}}^{2}$ can run to negative values at low energy, whereas, if $\lambda$ is not too large, $m_{H_{1}}^{2}$ remains positive. It seems also plausible that, for small $\lambda$ and $\mathrm{k}$ also $m_{N}^{2}$ remains positive, although a detailed resolution of the RGE's for the soft parameters would be necessary in order to clarify this point.

\footnotetext{
${ }^{2}$ Note [20] that in $\left(\mathrm{ReH}_{1}, \mathrm{Re} \mathrm{H}_{2}, \operatorname{ReN}\right)=\left(v_{1}, v_{2}, x\right)$ the point $\left(A^{0}, \operatorname{Im} N\right)=(0,0)$ is always a minimum, as the pseudoscalar mass matrix has only positive eigenvalues.
} 
However, in what follows, we will constrain the parameter space by means of eq. (6), requiring that the only negative soft mass is $m_{H_{2}}^{2}$.

The critical temperature will then be given, in any case, by

$$
T_{C} \approx T_{H_{2}}^{S}=\sqrt{-\frac{24 m_{H_{2}}^{2}+\Delta_{r a d}}{C_{H_{2}}}},
$$

where the $\approx$ is due to the fact that we have neglected the off-diagonal term in (16). Anyway, numerical results will be obtained by solving the condition (17) with the complete matrix.

\section{Minimization of the potential}

Once we have ensured the correct pattern of symmetry breaking, i.e. no VEV's for $\operatorname{Im} N, A^{0}$ and $C^{+}$(this last condition is guaranteed by eq. (7)) we can study the minimization of the potential, at the critical temperature, in the scalar directions only.

Before illustrating the numerical results of this minimization let us give an intuitive view of what actually happens, namely, of the crucial role played by the trilinear soft SUSY breaking parameters $A_{\lambda}$ and $A_{k}$ in the formation of a minimum of the effective potential for large values of the fields $\left(\sim T_{C}\right)$. We define the polar coordinates

$$
\left\{\begin{array}{l}
R e H_{1}^{0}=Y \cos \alpha \cos \beta^{T} \\
R e H_{2}^{0}=Y \cos \alpha \sin \beta^{T} \\
\operatorname{ReN}=Y \sin \alpha .
\end{array}\right.
$$

The full effective potential may then be written as the sum of terms of different order in $\tilde{Y} \equiv Y / T$

$$
\tilde{V} \equiv \frac{V}{T^{4}}=a \tilde{Y}+b \tilde{Y}^{2}+c \tilde{Y}^{3}+d \tilde{Y}^{4}+\cdots
$$

where the ellipses indicate terms coming from $\Delta V_{T=0}^{1-\text { loop}}$, which, in our approximation, are different from zero in the direction $H_{2}^{0}$ only and, due to the 
renormalization condition (10), will be small as long as we search a minimum at $T_{C}$ with $H_{2}^{0}$ of the same order of $v_{2}$.

The coefficients in (20) are given by:

i) linear terms:

$$
a=\frac{1}{8} \sin 2 \beta \sin \alpha \frac{\lambda A_{\lambda}}{T}
$$

ii) quadratic terms:

$$
b=\frac{1}{T^{2}}\left[\cos ^{2} \alpha\left(m_{H_{1}}^{2} \cos ^{2} \beta^{T}+m_{H_{2}}^{2} \sin ^{2} \beta^{T}\right)+m_{N}^{2} \sin ^{2} \alpha+\frac{T^{2}}{24}(\cdots)\right] ;
$$

here we do not write explicitly the terms coming from finite temperature corrections; for the present purposes what matters is that the complete quadratic term is positive or zero at $T=T_{C}$ in any direction;

iii) cubic terms:

$$
c=-\frac{1}{T} \sin \alpha\left(\lambda A_{\lambda} \cos ^{2} \alpha \sin 2 \beta^{T}+\frac{2}{3} k A_{k} \sin ^{2} \alpha\right) ;
$$

iv) quartic terms:

$$
\begin{aligned}
d & =\lambda^{2} \cos ^{2} \alpha\left(\sin ^{2} \alpha+\frac{1}{4} \cos ^{2} \alpha \sin ^{2} 2 \beta^{T}\right)+k^{2} \sin ^{4} \alpha \\
& -\frac{\lambda k}{4} \sin ^{2} 2 \alpha \sin 2 \beta^{T}+\frac{g^{2}+g^{\prime 2}}{8} \cos ^{4} \alpha \cos ^{2} 2 \beta^{T} .
\end{aligned}
$$

At large values of the fields, $\tilde{Y}^{2} \geq a / c(\sim O(1))$ (that is $Y \geq T_{C}$ ) the potential has a minimum at

$$
\tilde{Y} \approx-\frac{3 c}{4 d} ;
$$

the condition for this to be a global minimum is (neglecting the quadratic term)

$$
\left|\frac{c^{3}}{a d^{2}}\right| \sim \frac{\sin ^{2} \alpha\left(\lambda A_{\lambda} \cos ^{2} \alpha \sin 2 \beta^{T}+\frac{2}{3} k A_{k} \sin ^{2} \alpha\right)^{3}}{A_{\lambda} \sin 2 \beta T_{C}^{2} \lambda^{5}} \gg 1
$$

Assuming that eq.(21) is satisfied, the question is now to determine the direction of the vacuum in the field space. Since the only negative term 
appearing in (20) is the cubic one, and it is proportional to $\sin \alpha$, there will be always a $R e N$-component in the direction of the vacuum.

However, for large $A_{\lambda}$ the l.h.s. of (21) decreases for $\sin \alpha=1$ (it is the effect of the linear term that raises the potential in that direction), so that the $\sin \alpha \neq 0$ directions become favorite, and an electroweak first-order (nonzero VEV's for $\mathrm{ReH}_{1}, \mathrm{ReH}_{2}$ ) phase transition takes place.

\section{Numerical results.}

Contrary to the case of the Standard Model, the constraint (2) on the vacuum expectation value of the fields at the critical temperature cannot be written as a simple bound on the masses of the scalars in this model. In fact, approximate analytic expressions for these masses can be obtained [20] in the limiting cases $x \gg v_{1}, v_{2}$ or $x \ll v_{1}, v_{2}$, but we are here interested in values of $x$ of the same order of the electroweak VEV's $v_{1}, v_{2}$ ?. So we are induced to perform a numerical investigation on the parameter space in order to find the region in which the bound (2) is satisfied, and then look at the corresponding values for the mass of the lightest scalar.

In addition to the six tree-level parameters, we have to consider also those appearing in the one-loop corrections, namely, the top Yukawa coupling $h_{t}$, the stop soft SUSY breaking mass $\tilde{m}_{Q}^{2}$, and the gauginos direct masses $M_{1}$ and $M_{2}(14)$. We will assume a common value for $\tilde{m}_{Q}^{2}, M_{1}^{2}$ and $M_{2}^{2}$

$$
\tilde{m}_{Q}^{2}=M_{1}^{2}=M_{2}^{2}=\tilde{m}^{2}=1 T e V .
$$

Then we constrain $h_{t}, \lambda$, and $k$ by means of the renormalization group analysis performed by the authors of ref [25]. Requiring that the coupling $\lambda$ remains perturbative up to a large (say $10^{16} \mathrm{GeV}$ ) scale, they find, at $M_{Z}$

$$
\lambda^{2}\left(M_{Z}\right)<\frac{2 M_{Z}^{2}}{v^{2}}=0.274
$$

and

\footnotetext{
${ }^{3}$ One of the original reasons for the introduction of this model was the so called $\mu$ problem, whose solution requires $\lambda x \sim O\left(M_{W}\right)$ [20].
} 


$$
h_{t}\left(M_{Z}\right) \approx 0.97
$$

Moreover, RGE's for the couplings have the fixed ratio point (neglecting $g, g^{\prime}$ with respect to $h_{t}$ )

$$
\frac{k^{2}}{\lambda^{2}}=\frac{1}{2} .
$$

We will fix $\lambda$ at its upper value as given by (22) and $h_{t}$ and $k$ according to (23), (24).

For fixed $M_{2}$ and $\lambda$ the experimental limit [23] on the chargino mass gives a lower bound on $\mathrm{x}$, approximatively

$$
x \geq x_{\min } \approx \frac{45 \mathrm{GeV}}{\lambda} .
$$

We then fix $\tan \beta$ to some typical value (we will use $\tan \beta=2,10$ ) and require (see the discussion on the critical temperature)

$$
m_{H_{1}}^{2}>0, m_{H_{2}}^{2}<0 .
$$

This, in turn, implies

$$
A_{\lambda}^{\min }<A_{\lambda}<A_{\lambda}^{\max }
$$

where

$$
\begin{aligned}
& A_{\lambda}^{\min }=\frac{1}{x}\left[\lambda \operatorname{cotg} \beta\left(x^{2}+\frac{v^{2}}{4}\right)+\frac{\lambda v^{2}}{4} \sin 2 \beta-k x^{2}\right] ; \\
& A_{\lambda}^{\max }=\frac{1}{x}\left[\lambda t g \beta\left(x^{2}+\frac{v^{2}}{4}\right)+\frac{\lambda v^{2}}{4} \sin 2 \beta-k x^{2}\right],
\end{aligned}
$$

or, equivalently

$$
\frac{\lambda}{\sin ^{2} \beta}\left(x^{2}+\frac{v^{2}}{4}\right)<m_{c}^{2}<\frac{\lambda}{\cos ^{2} \beta}\left(x^{2}+\frac{v^{2}}{4}\right),
$$

where $m_{c}^{2}$ is the mass of the charged scalar. Eq. (26) automatically ensures that eq. (8) is satisfied, i.e., that the charged fields do not acquire any VEV.

Finally, the requirement $m_{N}^{2}>0$ implies a lower bound on $A_{k}$

$$
A_{k}>2 k x+\frac{\lambda v^{2}}{k x}\left[\lambda-\sin 2 \beta\left(\frac{A_{\lambda}}{2 x}+k\right)\right] .
$$


For any triplet $\left(x, A_{\lambda}, A_{k}\right)$ satisfying (25), (26), and (27), we minimize numerically the effective potential at zero temperature and verify that the vacuum is at $\left(\operatorname{Re} H_{1}^{0}, R e H_{2}^{0}, \operatorname{ReN}\right)=\left(v_{1}, v_{2}, x\right)$. We then calculate the critical temperature $T_{C}$ according to the definition (17), and, finally, minimize the effective potential at $T_{C}$.

In Fig. 1 we report $v\left(T_{c}\right) / T_{c}$ against the lightest scalar $\left(m_{S_{1}}\right)$ mass for all the points that passed the above mentioned selections. As we see there are a lot of points corresponding to $v\left(T_{c}\right) / T_{c}>1.3$ and $m_{S_{1}}>60 \mathrm{GeV}$. Note that the maximum allowed values for $m_{S_{1}}$ reach $170 \mathrm{GeV}$.

In Figs. 2a, 2b we plot the allowed region (i.e. the points corresponding to $\left.v\left(T_{c}\right) / T_{c}>1.3\right)$ in the $m_{S_{1}}-m_{c}$ plane, where $m_{c}$ is the charged scalar mass; $x$ is fixed at $300 \mathrm{GeV}$, while the upper and lower limits for $m_{c}$ are given by (26).

Finally, in Fig. 3a, 3b we recover the result we got from the naive discussion on the minimization of the potential, namely, the crucial role played by $A_{\lambda}$ in the determination of the direction of the transition. The dashed lines represent the values of $v\left(T_{C}\right)$, while the continuos ones those of $T_{C}$.

In conclusion, we have showed explicitly that minimal extensions of the MSSM allow to easily circumvent the Higgs mass problem arising from the study of the electroweak phase transition in the Standard Model and in the MSSM. The main role is played by the trilinear soft SUSY breaking terms, which are present in any supersymmetric model with extra SU(2)-singlet superfields. Radiative effects are also important as, due to the heavy top, they force the direction of the transition (i.e. the flat direction of the potential at $\left.T_{C}\right)$ to be always $\mathrm{ReH}_{2}$.

\section{Acknowledgments}

The author would like to thank A. Masiero, who inspired this work and followed each stage of its preparation, and G.F. Giudice, who read the manuscript and provided helpful comments and suggestions. Useful discussions with D. Comelli, F. Illuminati and F. Zwirner are also acknowledged. 


\section{References.}

[1] A.D. Sakharov, Pis'ma Zh. Eksp. Teor. Fiz. 5 (1967) 32, JETP Lett. 5 24 (1967)

[2] A.Yu. Ignatiev, N.V. Krashnov, V.A. Kuzmin and A.N. Tavkhelidze, Proc. Int. Conf. Neutrino '77 Vol.2 (Nauka, Moskow,1978) p.293, Phys. Lett. B76 (1978) 436;

M. Yashimura, Phys. Rev. Lett. 41(1978) 281, 42 (1979) 476 (E);

S. Weinberg, Phys. Rev. Lett. 42(1979) 850;

A.Yu. Ignatiev, V.A. Kuzmin and M.E. Shaposhnikov, Phys. Lett. B87 (1979) 114

[3] V.Kuzmin, V.Rubakov and M. E. Shaposnikov, Phys. Lett. B155 ( 1985) 36

[4] J.S. Harvey and M.S. Turner, Phys. Rev. D42 (1990) 3344

[5] B. Campbell, S. Davidson, J. Ellis and K.A. Olive, Phis. Lett. B256 (1991) 457;

W.Fishler, G.F. Giudice, R.G. Leigh and S.Paban, Phys. Lett B258 (1991) 45;

K. Enqvist, A. Masiero and A. Riotto, Nucl. Phys. B373 (1992) 95

[6] A.D. Dolgov, YITP/K 90 preprint (1991) submitted to Phys. Rep.; 

417

A.I. Bochkarev and M.E. Shaposhnikov, Mod. Phys. Lett. A6(1987)

A.I. Bochkarev, S. Kuzmin and M.E. Shaposhnikov, Phys. Lett. B244 (1990) 275;

A.I. Bochkarev, S. Kuzmin and M.E. Shaposhnikov, Phys. Rev. D43 (1991) 369;

[7] M.E. Shaposhnikov, Nucl.Phys B287 (1987) 757

[8] N.Turok and J.Zadrozny, Nucl. Phys B369 (1992) 729

[9] G.W. Anderson and L.J. Hall, Phys. Rev. D45 (1992) 2685

[10] G.F. Giudice, preprint UTTG-35-91

[11] M.Dyne, R.G. Leigh, P.Huet, A. Linde and D. Linde SLAC-PUB-5740, SLAC-PUB-5741

[12] A.Masiero and A.Riotto, University of Padova preprint DFPD /92/TH/22

[13] M.Dine, P.Huet and R.Singleton Jr. Nucl. Phys B375 (1992) 625

[14] J. I. Kapusta, Finite temperature field theory (Cambridge University Press, 1989)

[15] L.Dolan and R.Jackiw, Phys. Rev. D9 (1974) 3320;

S.Weinberg, Phys. Rev. D9 (1974) 3357

[16] D.A. Kirznhits and A.D. Linde, Ann. Phys. 101 (1976) 195; 
A.D. Linde, Rep. Progr. Phys. 42 (1979) 389;

K.Takahashi, Z.Phys. C26 (1985) 601;

D.E. Brahm, S.D.H. Hsu, preprint CALT-68-1705;

M.E. Carrington, preprint TPI-MINN-91/48-T;

M.E. Shaposhnikov, CERN-TH 6319/91

[17] M. Davier in Proc. of the Lepton-Photon Symposium and Europhysics Conference

on High Energy Physics, S. Hagerty, K. Petter and E. Quercigh (eds.) (Geneva, 1991)

[18] K. Enqvist, K. Kainulainen and I.Vilja,preprint NORDITA 92/10 P

[19] J.P. Derendinger and C.A. Savoy, Nucl. Phys. B237 (1984) 307

[20] J.Ellis, J.F. Gunion, H.E. Haber, L.Roszkowsky and F.Zwirner,

Phys. Rev. D39 (1983) 844

[21] J.Ellis, K.Enqvist, D.V. Nanopoulos, K. Olive, M. Quiros and F. Zwirner, Phys. Lett. B176 (1986) 403

[22] J. Ellis, G. Ridolfi, F. Zwirner, Phys. Lett. B262 (1991) 477

[23] ALEPH collaboration, preprint CERN-PPE/91-149

[24] A.D. Linde, Nucl. Phys. B216 (1983) 421, Phys. Lett. B100 (1981) 137 
[25] P.Binéutruy, C.A. Savoy, Phys. Lett. B277 (1992) 453 


\section{Figure Captions.}

Fig. $1 v\left(T_{C}\right) / T_{C}$ ratio vs. the lightest scalar mass, for all the allowed region for the parameters $\lambda, \mathrm{k}, \mathrm{x}, A_{\lambda}$, and $A_{k}$ (see text), and $\operatorname{tg} \beta=2$.

Fig. 2a Upper and lower bounds for the lightest scalar mass coming from the various conditions imposed on parameter space (see text) plus the requirement that $\left.v\left(T_{C}\right) / T_{C}\right)>1 / 3$. x has been fixed to $300 \mathrm{GeV}$ and $\operatorname{tg} \beta=2$. Fig. 2 b The same as in fig 2 a with $\operatorname{tg} \beta=10$.

Fig. 3a $v\left(T_{C}\right)$ and $T_{C}$ dependence from $A_{\lambda}$, for $x=300 \mathrm{GeV}, A_{k}=190 \mathrm{GeV}$ and $\operatorname{tg} \beta=2$.

Fig. 3b Same as in Fig. 3a for $x=400 \mathrm{GeV}, A_{k}=300 \mathrm{GeV}$ and $\operatorname{tg} \beta=10$. 